\title{
Histopathologic and clinical comparison of recurrent and non-recurrent urethral stricture disease treated by reconstructive surgery
}

\author{
Iryna V. Samarska ${ }^{1,2} \wedge$, Hasan Dani ${ }^{3}$, Trinity J. Bivalacqua ${ }^{3,4}$, Arthur L. Burnett ${ }^{3,4}$, Andres Matoso ${ }^{2,3,4} \wedge$ \\ ${ }^{1}$ Department of Pathology, Maastricht University Medical Center, Maastricht, The Netherlands; ${ }^{2}$ Department of Pathology, The Johns Hopkins \\ Medical Institutions, Baltimore, MD, USA; ${ }^{3}$ Department of Urology, The Johns Hopkins Medical Institutions, Baltimore, MD, USA; ${ }^{4}$ Department \\ of Oncology, The Johns Hopkins Medical Institutions, Baltimore, MD, USA \\ Contributions: (I) Conception and design: A Matoso; (II) Administrative support: IV Samarska, A Matoso; (III) Provision of study materials or \\ patients: IV Samarska, H Dani, AL Burnett, A Matoso; (IV) Collection and assembly of data: IV Samarska, H Dani, A Matoso; (V) Data analysis and \\ interpretation: IV Samarska, H Dani, A Matoso; (VI) Manuscript writing: All authors; (VII) Final approval of manuscript: All authors. \\ Correspondence to: Andres Matoso, MD. Department of Pathology, Johns Hopkins Hospital, Weinberg 2242, 401 N. Broadway, Baltimore, MD 21231, \\ USA. Email: amatoso1@jhmi.edu.
}

\begin{abstract}
Background: Urethral stricture is a relatively frequent problem often requiring multiple surgical interventions. The objective of this study was to compare the clinicopathologic features of urethral resections from patients who underwent open end-to-end anastomotic urethroplasty and later recurred compared to those who did not.
\end{abstract}

Methods: A retrospective review of the pathology files identified 36 consecutive patients who underwent urethroplasty. The histopathological analysis included evaluation of the inflammatory infiltrate based on the predominant $(>50 \%)$ cell type: lymphocyte-rich, neutrophil-rich, plasma cell-rich, and mixed; length and thickness of the fibrous plaque; and the cellularity of the fibrous plaque: cellular ( $>40$ stroma nuclei/HPF) or paucicellular (<40 stroma nuclei/high power field).

Results: Ten (28\%) patients recurred, and 26 (72\%) did not. There was no significant difference between recurrent and non-recurrent cases in age, race, comorbidities, location of the stricture, and etiology. All patients with recurrent strictures showed dense paucicellular fibrotic plaques (10/10; 100\%), while this was seen in $14 / 26(53.8 \%)$ non-recurrent cases $(\mathrm{P}=0.01)$. Only one patient with cellular fibrosis showed recurrence during follow-up. The log-rank test shows that time to recurrence is significantly shorter in patients with paucicellular fibrosis compared to those with cellular fibrosis $(\mathrm{P}=0.036)$. The inflammation consisted of a mixed population of CD3(+) T-lymphocytes, CD20(+) B-lymphocytes, and CD68(+) histiocytes, and there was no difference in the composition of the inflammation between groups. All cases with plasma cell-rich infiltrate showed normal IgG4:IgG.

Conclusions: Our study supports reporting cellularity of the fibrous plaque as a potential predictor of outcome in patients undergoing reconstructive urethroplasty. Patients with paucicellular fibrosis are at increased risk of recurrence.

Keywords: Urethral stricture; urethroplasty; urethral obstruction

Submitted May 28, 2021. Accepted for publication Aug 26, 2021.

doi: 10.21037/tau-21-477

View this article at: https://dx.doi.org/10.21037/tau-21-477

^ ORCID: Andres Matoso, 0000-0003-0990-1115; Iryna V. Samarska, 0000-0003-4092-0672; Arthur L. Burnett, 0000-0001-6560-8605. 


\section{Introduction}

Urethral stricture is a relatively frequent problem in urological practice with a relative comparable incidence of $0.6 \%$ of all urologic consults in the United States and other developed countries $(1,2)$. The etiology includes idiopathic (unknown), iatrogenic (post-instrumental), congenital (hypospadias-related), inflammatory (sexually transmitted diseases-related), traumatic (pelvis fractures) and lichen sclerosus-related (formerly known as balanitis xerotica obliterans) (3-6). Idiopathic or unknown cause is the most common etiology in both bulbar and penile strictures $(6,7)$.

The pathophysiology of urethral strictures is mostly unknown. Strictures may result due to ischemic spongiofibrosis occurring after infective, inflammatory, or traumatic injury $(3,7)$. The pathogenesis includes excessive fibroblast proliferation, collagen synthesis, and extracellular matrix deposition (8). Recently IgG4-related disease was proved to be an etiological factor in ureteral stenosis (9), and BK-virus infection has been shown in relation to both ureteral and urethral stenosis (10).

The aim of this study is to describe the clinicopathologic features of the urethral stricture resections from patients who later recurred compared to those who did not recur on follow-up. We hypothesized that the pattern of inflammation and fibrosis pattern could be used to predict the outcome. We present the following article in accordance with the STROBE reporting checklist (available at https:// dx.doi.org/10.21037/tau-21-477).

\section{Methods}

\section{Patients}

The study was conducted in accordance with the Declaration of Helsinki (as revised in 2013). The study was approved by institutional review board of Johns Hopkins University (No. IRB00254688) and individual consent for this retrospective analysis was waived. We identified 36 consecutive patients with urethral stricture treated with surgical excision from the files of Department of Pathology of Johns Hopkins Medical Institutions in a period of 3 years. Since the goal of the study was to determine histopathologic predictors of recurrence, patients operated with flaps or grafts were excluded to decrease confounders. Only patients with available histopathological material were included. Patients with a history of radiation therapy to the pelvis were also excluded. A retrospective review was performed to collect patient demographics and history of infection, trauma, previous urological procedures, preand postoperative urodynamic characteristics, and evaluate the postoperative value of the international symposium of prostate score (IPSS) and the sexual health inventory for men (SHIM) score. The non-recurrent strictures were defined as the first diagnosis with successful treatment during the documented follow-up period without requiring additional urological intervention. The recurrent strictures were classified as patients requiring internal urethrotomy and/or intermittent dilation. Surgical success was defined as absence of symptoms of lower urinary tract obstruction (LUTS) and no instrumentation after the resection. The status of recurrent or non-recurrent is at the time of last follow-up visit. When no anamnestic or documented cause was identified, the stricture was classified as idiopathic.

\section{Histopathology and immunobistochemistry}

Tissue samples were totally submitted for the histopathological evaluation. All tissue samples were first fixed in buffered formalin and processed to paraffin wax. The 3-4 um sections were stained with hematoxylin and eosin (H\&E) for histologic evaluation. The histopathological analysis included assessing the inflammatory infiltrate based on the predominant $(>50 \%)$ cell type, including lymphocyte-rich, neutrophil-rich, plasma cell-rich, and mixed infiltrate. The cellularity of the fibrous area was measured as a number of the stromal cells in 5 HPFs ( $\times 40$ magnification) in the areas with the maximal stromal cellularity. Nuclei of inflammatory cells were not counted. The cellularity of the fibrous area was classified as cellular ( $>40$ stroma nuclei/HPF) or paucicellular ( $<40$ stroma nuclei/HPF), adapted from the previously published studies and confirmed by the number of the stromal cells in the cases with cellular fibrosis from this study $(11,12)$. Trichrome-blue stain (Masson's trichrome stain) was performed to evaluate the extent of fibrosis below the urothelium or urethral lumen by measuring the thickness of deep blue staining in $\mathrm{mm}$ perpendicular to the lumen. The fibrotic plaque was defined as a dense fibrotic tissue, characterized by the packs of collagen fibers, strongly positive for Trichromeblue stain, as previously described (11). In the samples with a prominent lymphoplasmacytic inflammation (lymphocyte rich cases), immunohistochemistry for CD3, CD20, CD68, $\mathrm{IgG}$, and IgG4 was performed. Immunohistochemistry was performed using the Ventana Medical System Benchmark XT Ultra (Ventana, Tucson, AZ, USA) with the following 
specific antibodies: anti-CD20 (clone MS/L26, Ventana; predilute), anti-CD3 (Dako polyclonal rabbit anti-human CD3; 1:00 dilution) anti-CD68 (clone KP-1, Ventana; predilute), anti-IgG (NCL-L-IgG, Leica Biosystems, Buffalo Grove, IL, USA; 1:500 dilution), and anti-IgG4 (catalog \#A-10651; ThermoFisher Scientific, Waltham, MA, USA; 1:2,000 dilution). The number of positive cells was manually counted in 5 HPFs in hotspot and averaged. All samples were reviewed and measured by two pathologists (IS and $\mathrm{AM}$ ) blinded to the recurrence/non-recurrence status of the patient.

\section{Statistical analysis}

Statistical analysis was performed using GraphPad Prism 9 (GraphPad Software, San Diego, CA, USA). The p values were calculated using the student's $t$-test and chi-square test. Results were reported in mean \pm SD. Statistical significance was considered if $\mathrm{P} \leq 0.05$ and $\mathrm{P}$ values $>0.05$ were reported as not significant (NS).

\section{Results}

\section{Clinicopathologic findings}

We identified 36 consecutive male patients who underwent reconstructive surgery and with a segment of urethral stricture excised and available for histologic studies. The surgical procedure performed was open urethroplasty with end-to-end anastomosis. Follow-up information determined that $26(72 \%)$ patients were non-recurrent and 10 (28\%) were recurrent. The average follow-up time was 68.2 months for the recurrent group (range, 28-184 months) and 53.0 months for the nonrecurrent group (range, $25-134$ months). The median time to recurrence was 15 months (range, 2-79 months), but 8/10 $(80 \%)$ recurred within the first year after the urethroplasty, with a median time to recurrence of 4 months.

Age, race, etiology, stricture location, and the stricture size were similar in recurrent and non-recurrent groups (Table 1).

The average IPSS, which measures lower urinary tract symptoms, was 17.4 in the non-recurrent group and 22.14 in the recurrent group $(\mathrm{P}=0.09)$. The average SHIM score, which measures erectile function, was 14.4 for nonrecurrent patients and 11.6 for recurrent patients $(\mathrm{P}>0.05)$.

Seventeen patients $(65.5 \%)$ in the non-recurrent group and $9(90 \%)$ in the recurrent group had at least one direct vision internal urethrotomy (DVIU) or dilatation procedure prior to the urethroplasty $(\mathrm{P}>0.05)$. Three patients $(11.5 \%)$ of the non-recurrent patients and $2(20 \%)$ of the recurrent patients have had a previous urethroplasty $(\mathrm{P}>0.05)$ in a different location, and therefore, the current resection was not considered a recurrence. None of the patients had a pre-operative Foley catheter in either recurrent or non-recurrent group. One patient in each group was on intermittent catheterization prior to the resection and endto-end anastomosis.

The average baseline voided volume was $309 \mathrm{~mL}$ in the non-recurrent group and $367 \mathrm{~mL}$ in the recurrent group $(\mathrm{P}>0.05)$. The post urethroplasty voided volume was $417 \mathrm{~mL}$ in the non-recurrent group and $457 \mathrm{~mL}$ in the recurrent group $(\mathrm{P}>0.05)$. The presurgical uroflow was $9.95 \mathrm{~mL} / \mathrm{s}$ in the non-recurrent group and $11.66 \mathrm{~mL} / \mathrm{s}$ in the recurrent group $(\mathrm{P}>0.05)$. The post urethroplasty uroflow was $28.89 \mathrm{~mL} / \mathrm{s}$ in the non-recurrent group and $11.67 \mathrm{~mL} / \mathrm{s}$ in the recurrent group $(\mathrm{P}=0.001)$.

\section{Histopathology}

Cases with non-recurrent stricture showed paucicellular fibrosis in 14/26 (53.8\%, Figure 1) and cellular fibrosis in $12 / 26$ (46.2\%, Figure 2). In the cases with cellular fibrosis the average number of stromal cells was $42.8 \pm 3.0$ per HPF. Twelve patients (50\%) with the non-recurrent disease had associated inflammation in the area of fibrosis, including lymphocyte-rich infiltrate in $11 / 26(42.3 \%)$, and plasma cell-rich infiltrate in $1 / 26(3.8 \%)$. All patients with recurrent urethral strictures showed paucicellular fibrotic plaques $(10 / 10 ; 100 \%)$, defined as dense fibrotic tissue, characterized by the packs of collagen fibers, strongly positive for Trichrome-blue stain. The average number of stromal cells was $12 \pm 1.8$ per HPF in this group. The fibrous plaque in the recurrent disease was associated with inflammation in $3 / 10(30 \%)$ patients, including lymphocyte rich infiltrate in $2 / 10(20 \%)$ and plasma cell rich in $1 / 10(10 \%)$. The inflammation showed a mixed infiltrate consistent with a reactive process composed of a mixed population of CD3(+) T-lymphocytes, CD20(+) B-lymphocytes, and CD68(+) histiocytes. The number of $\mathrm{IgG}(+)$ plasma cells varied greatly between cases; however, all cases showed a normal IgG4:IgG ratio $(<10 \%)$. There was no difference between the groups in the thickness of fibrotic area measured in slides stained with trichrome blue $(0.92 \pm 1.2$ and $0.97 \pm 1.4 \mathrm{~mm}$, in recurrent and nonrecurrent groups, respectively). Table 2 summarizes the histopathologic findings. The log-rank test for time to 
Table 1 Clinicopathologic features of patients

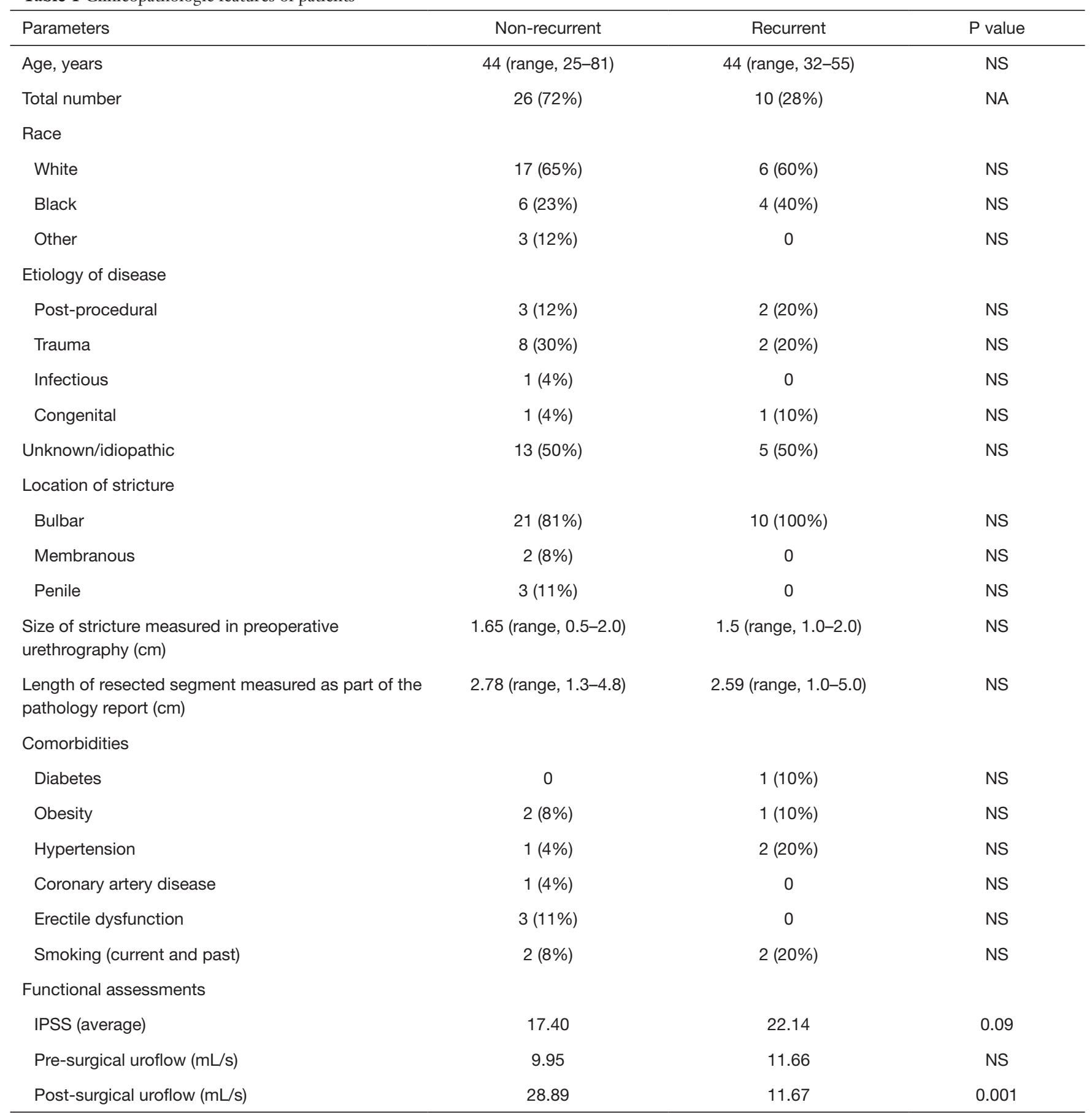

NS, not significant, $\mathrm{P}$ values $>0.05$; NA, not applicable/not available; IPSS, international symposium of prostate score.

recurrence shows a significantly shorter time to recurrence in patients with paucicellular fibrosis compared to patients with loose fibrosis $(\mathrm{P}=0.036)$ and no difference between patients with and without inflammation $(\mathrm{P}=0.47$, Figure 3).

\section{Discussion}

Urethral stricture disease requiring open urethroplasty has a negative impact on the quality of life of patients and is an important economic burden for the health care systems. 

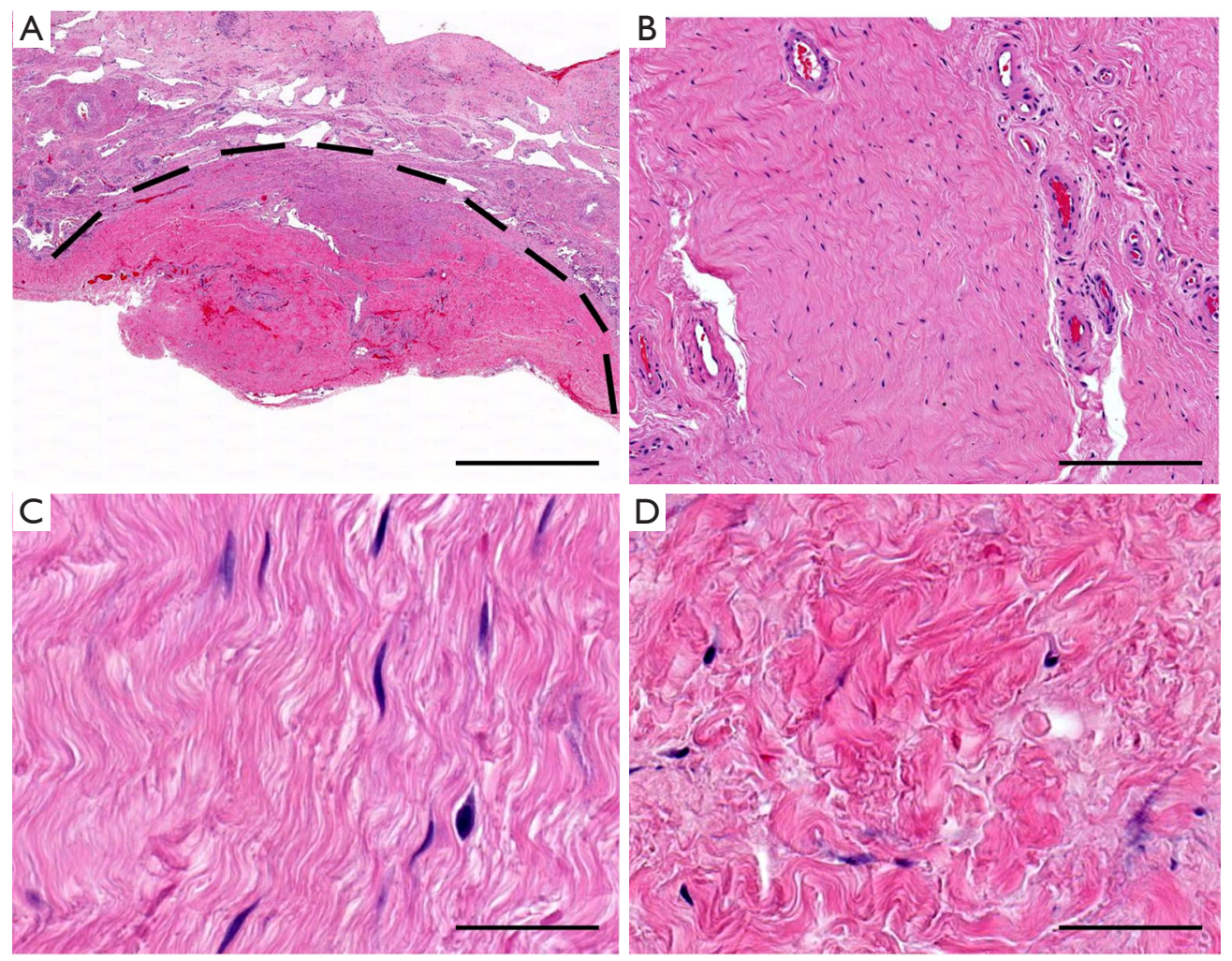

Figure 1 Histologic findings in urethral stricture with dense fibrosis. (A) Whole slide cross section of area of stricture. The area under the dotted line is the scared tissue. (Hematoxylin-eosin; scale bar $2 \mathrm{~mm}$ ). (B) Higher power of (A) in the area of fibrosis shows scattered stromal nuclei. (Hematoxylin-eosin stain; scale bar $200 \mu \mathrm{m}$ ). (C,D) High power view of area of fibrosis with scattered stromal nuclei. Note the nuclei are elongated and surrounded by multiple layers of dense collagen deposition. (Hematoxylin-eosin stain; scale bar $100 \mu \mathrm{m}$ lower left and $30 \mu \mathrm{m}$ lower right).

Patients with this disease suffer symptoms of LUTS, including detrusor hypertrophy, diverticular bladder disease, urinary infections, urinary stones, and renal failure $(7,13,14)$. Reconstructive surgery is the alternative for patients who fail DVIU or with obstructions larger than $1 \mathrm{~cm}$. Open urethroplasty has a high success rate estimated to be around or higher than $75 \%$. However, many patients still present with obstructive symptoms requiring continuous followup with uroflowmetry, dilatations, urethrotomy, or even repeat urethroplasty $(15,16)$. The care of these patients is estimated at several hundred million U.S. dollars per year $(13,17)$. Therefore, determining which patients are at increased risk of recurrence has been the subject of multiple studies. However, a significant problem comparing results of different studies, is that there are no well-established guidelines outlining the surveillance protocols for patients who have undergone urethral reconstruction surgery (18). Additionally, it is difficult to compare outcomes of studies with a mixed population of patients, performed at different institutions, and with strictures of different urethral portions (17). Our study is a single institution study including patients operated with only one technique (end primary anastomosis) and almost exclusively bulbar urethral cases, limiting some of the variables with potential impact in outcomes and allows for a better comparison between recurrent and non-recurrent disease.

In this study, we investigated specific histopathologic features of the fibrous plaque removed during urethroplasty in patients undergoing end-to-end anastomosis. The features studied here are routine morphologic assessments, including the thickness of the fibrous area, the cellularity of the stroma, and the composition of the inflammatory response in cases with marked inflammation. Cases with non-recurrent stricture showed both hypocellular and cellular fibrosis. In contrast, all patients with recurrent urethral strictures showed paucicellular fibrotic plaques, 

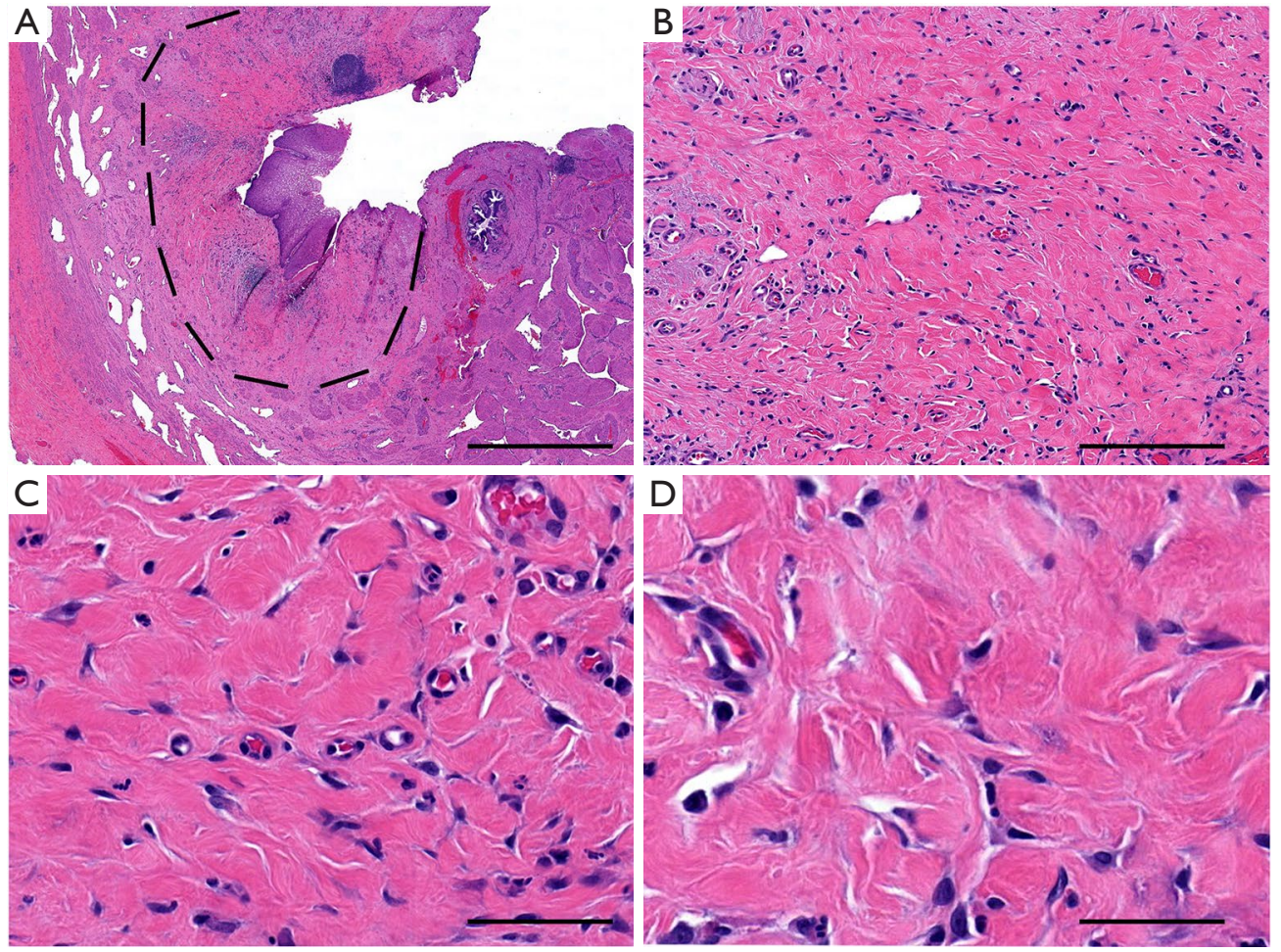

Figure 2 Histologic findings in urethral stricture with loose fibrosis. (A) Whole slide cross section of area of stricture. The area within the dotted line is the scared tissue. The urothelium shows squamous metaplasia in the area overlying the stricture. (Hematoxylin-eosin; scale bar $2 \mathrm{~mm}$ ). (B) Higher power of (A) in the area of fibrosis shows scattered stromal nuclei (Hematoxylin-eosin stain; scale bar 200 mm). (C) Higher power of (A) in the area of fibrosis shows frequent stromal nuclei. Note the stromal nuclei are more rounded than the nuclei of the fibrosis of "dense fibrous plaques" shown in Figure 1. (Hematoxylin-eosin stain; scale bar $100 \mu \mathrm{m}$ ). (D) High power of (A) showing area of fibrosis with frequent nuclei. In areas, the stromal nuclei appear to come in contact with one-another, a feature not seen in dense fibrous plaques. (Hematoxylin-eosin stain; scale bar $200 \mu \mathrm{m}$ ).

Table 2 Fibrosis and inflammatory infiltrate analysis.

\begin{tabular}{lccc}
\hline Parameter & Recurrent $(\mathrm{n})$ & Non-recurrent $(\mathrm{n})$ & $\mathrm{P}$ value \\
\hline Paucicellular fibrotic plaque & $10 / 10(100 \%)$ & $14 / 26(53.8 \%)$ & 0.01 \\
Thickness of fibrosis $(\mathrm{mm})$ & $0.92 \pm 1.20$ & $0.97 \pm 1.40$ & $\mathrm{NS}$ \\
Inflammatory infiltrate & $2 / 10(20 \%)$ & $12 / 24(50 \%)$ & $\mathrm{NS}$ \\
CD3 & $60.00 \pm 16.82$ & $45.26 \pm 21.60$ & $\mathrm{NS}$ \\
CD20 & $49.2 \pm 38.0$ & $46.5 \pm 68.8$ & $\mathrm{NS}$ \\
CD68 & $14.7+/ 8.8$ & $13.4 \pm 5.6$ & $\mathrm{NS}$ \\
$\operatorname{lgG}$ /lgG & $0.17 \pm 0.06$ & $0.36 \pm 0.07$ & $\mathrm{NS}$ \\
\hline
\end{tabular}

NS, not significant; $P$ values >0.05; IgG4, immunoglobulin G4; IgG, immunoglobulin G.

as indicated by the low number of stromal cells in the area of the stricture. We did not observe a difference in the thickness or extent of the fibrotic area between groups suggesting that these are not as predictive of recurrence as cellularity is. It is possible that paucicellular plaques further affect the flexibility of the urethra and are, 

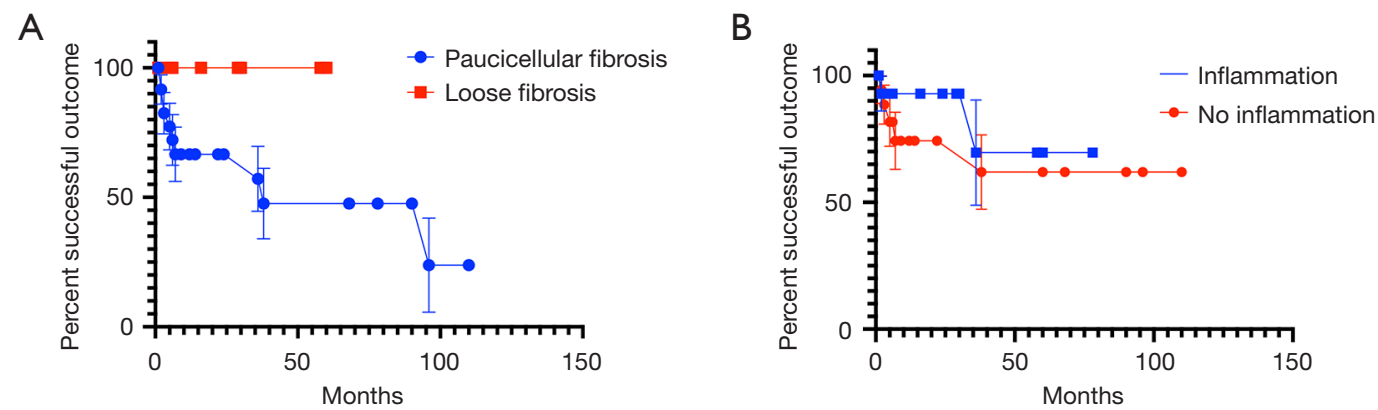

Figure 3 Kaplan-Meier analysis of time to recurrence. (A) Recurrence is only seen in patients with dense fibrous plaques (P=0.04). (B) There was no difference in time to recurrence in patients with and without inflammation.

therefore, associated with more symptoms of obstruction. Additionally, the cellularity of the fibrous plaque decreases as more collagen is deposited in the extracellular matrix. Consequently, it is likely indicative of the type of scar tissue that a given patient is predisposed to form.

We established a cut-off for cellularity of the fibrous area at $<40$ stroma nuclei/HPF. This limit was determined to achieve the highest possible specificity for paucicellular fibrosis as a predictor of recurrence. This could potentially spare patients without paucicellular fibrosis from frequent follow-up visits, should these findings be validated by further studies with more patients. All patients with recurrent strictures showed dense paucicellular fibrotic plaques, while this was seen in $53.8 \%$ non-recurrent cases.

We did not find an association between inflammation and recurrence rate. Similarly, a recently published study with emphasis on the histopathologic findings of anterior urethral strictures did not report the difference in recurrence rates based on inflammation (12). Furthermore, in cases with significant lymphoplasmacytic infiltration, we did not observe a high enough $\operatorname{IgG}$ : $\mathrm{IgG}$ ratio to support IgG4-related disease as a potential etiologic factor.

Two studies by Breyer et al. identified smoking, obesity, diabetes mellitus, prior DVIU, prior urethroplasty, and stricture length $>4 \mathrm{~cm}$ as risk factors associated with recurrence $(19,20)$. A study of patients with bulbar urethroplasty also found a correlation between body mass index (BMI) higher than 35 and recurrence (21). Another study by Yalcinkaya et al. did not find an association between recurrence and etiologic factors or age but identified a direct correlation between the size of stricture and worse overall outcome (22). Among the clinical and epidemiologic data collected, we did not observe any association with recurrence. However, we acknowledge that our study is underpowered to identify significant epidemiologic differences between groups.

Patients with recurrence disease were, on average, of the same age as patients with the non-recurrent disease. Several studies have previously reported that age negatively impacts outcomes of patients with urethral stricture disease undergoing urethroplasty. A multi-institutional study by Levy et al. compared the clinical characteristics of patients 60 years old or younger and older than 60 years old. The investigators identified that patients older than 60 years of age have significantly more comorbidities, including coronary artery disease, hypertension, hyperlipidemia, and cancer, than younger patients but did not find any difference in 3 months and 1-year functional outcomes between groups (23). However, a follow-up of only one year is insufficient to detect later symptomatic recurrence. In our study, we only had four patients who were older than 60 years old, including 1 with recurrent disease and 3 with non-recurrent stricture, which is insufficient to arrive at any conclusion as how age could impact the outcome. While there are multiple reports suggesting older individuals have a worse outcome after urethroplasty, these are thought to be related to a higher rate of comorbidities and worse blood supply. The current American Urologic Association (AUA) guidelines emphasize that an individual with recurrent urethral stricture is unlikely to result in a durable response if treated with minimally invasive techniques, including dilatation or DVIU. The guidelines do not support the use of age as a criterium to decide eligibility for urethroplasty, and therefore, the decision is left to the surgeon on a caseby-case approach.

Notably, the recurrent group did not show an improvement of the postoperative uroflow compared to the pre-surgical measure. This is markedly different to the non-recurrence 
group that did experience significant increase in the uroflow post-surgically. This raises the possibility that the stricture was not completely removed during the excision. A follow-up study analyzing only patients with significant improvement in the uroflow could help to better determine the importance of histologic evaluation in predicting recurrence.

There are several notable limitations of this study. This was an exploratory, hypothesis generating study, that needs to be confirmed with larger number of patients and prospectively collected. A prospective collection would potentially improve the follow-up time. Almost half of the patients from the non-recurrent group also had paucicellular fibrotic plaque, and they may potentially have recurred if the follow-up was longer. Additionally, this study does not include histopathology of the normal urethra adjacent to stricture site and/or information on the resection margins. It is unclear whether fibrosis and/or inflammation, or their absence, have an impact on the postsurgical outcome. Our study showed a significant difference between two groups, since all patients with recurrent strictures and only $53.8 \%$ non-recurrent cases showed dense paucicellular fibrotic plaques. However, it is not clear whether the same results would be achieved in a larger cohort. Moreover, the occurrence of dense paucicellular fibrotic plaques should be studied in all types of urethroplasty. Therefore, a prospective study with a larger number of patients is necessary to improve methodological approach and test the hypothesis.

\section{Conclusions}

Our study suggests that the reporting of the cellularity of the urethral fibrosis and paucicellular fibrous plaques in the specimen of the urethral stricture could represent a potential predictor of symptomatic recurrence in patients undergoing reconstructive urethroplasty. Timely reporting of this easy-to-obtain finding can help postoperative management and follow-up. However, further research is necessary to validate this finding.

\section{Acknowledgments}

Funding: This study was supported by the Department of Pathology at Johns Hopkins Hospital.

\section{Footnote}

Reporting Checklist: The authors have completed the STROBE reporting checklist. Available at https://dx.doi. org/10.21037/tau-21-477

Data Sharing Statement: Available at https://dx.doi. org/10.21037/tau-21-477

Peer Review File: Available at https://dx.doi.org/10.21037/ tau-21-477

Conflicts of Interest: All authors have completed the ICMJE uniform disclosure form (available at https://dx.doi. org/10.21037/tau-21-477). ALB reports consulting fees from Astellas, from Comphya, from Futura Medical, from Lilly, LLC, from Myriad Genetics, from Novartis Pharmaceuticals, from Reflexonic, LLC, and from Urology Times Editorial Council, outside the submitted work, and leadership position in UroMissionsWorks. The other authors have no conflicts of interest to declare.

Ethical Statement: The authors are accountable for all aspects of the work in ensuring that questions related to the accuracy or integrity of any part of the work are appropriately investigated and resolved. The study was conducted in accordance with the Declaration of Helsinki (as revised in 2013). The study was approved by institutional review board of Johns Hopkins University (NO. IRB00254688) and individual consent for this retrospective analysis was waived.

Open Access Statement: This is an Open Access article distributed in accordance with the Creative Commons Attribution-NonCommercial-NoDerivs 4.0 International License (CC BY-NC-ND 4.0), which permits the noncommercial replication and distribution of the article with the strict proviso that no changes or edits are made and the original work is properly cited (including links to both the formal publication through the relevant DOI and the license). See: https://creativecommons.org/licenses/by-nc-nd/4.0/.

\section{References}

1. Alwaal A, Blaschko SD, McAninch JW, et al. Epidemiology of urethral strictures. Transl Androl Urol 2014;3:209-13.

2. Hillary CJ, Osman NI, Chapple CR. Current trends in urethral stricture management. Asian J Urol 2014;1:46-54.

3. Hong MKH, Murugappan S, Norton SM, et al. Male urethral stricture disease in a regional centre: 10 years of experience. ANZ J Surg 2019;89:747-51.

4. Levy A, Browne B, Fredrick A, et al. Insights into the 
Pathophysiology of Urethral Stricture Disease due to Lichen Sclerosus: Comparison of Pathological Markers in Lichen Sclerosus Induced Strictures vs Nonlichen Sclerosus Induced Strictures. J Urol 2019;201:1158-63.

5. Levy AC, Moynihan M, Bennett JA, et al. Protein Expression Profiles among Lichen Sclerosus Urethral Strictures-Can Urethroplasty Success be Predicted? J Urol 2020;203:773-8.

6. Lumen N, Hoebeke P, Willemsen P, et al. Etiology of urethral stricture disease in the 21st century. J Urol 2009;182:983-7.

7. Cotter KJ, Hahn AE, Voelzke BB, et al. Trends in Urethral Stricture Disease Etiology and Urethroplasty Technique From a Multi-institutional Surgical Outcomes Research Group. Urology 2019;130:167-74.

8. Cavalcanti AG, Costa WS, Baskin LS, et al. A morphometric analysis of bulbar urethral strictures. BJU Int 2007;100:397-402.

9. Marando A, D'Ambrosio G, Catanzaro F, et al. IgG4related disease of the ureter: report of two cases and review of the literature. Virchows Arch 2013;462:673-8.

10. Rajpoot DK, Gomez A, Tsang W, et al. Ureteric and urethral stenosis: a complication of BK virus infection in a pediatric renal transplant patient. Pediatr Transplant 2007;11:433-5.

11. Da Silva EA, Schiavini JL, Santos JB, et al. Histological characterization of the urethral edges in patients who underwent bulbar anastomotic urethroplasty. J Urol 2008;180:2042-6.

12. Grimes MD, Tesdahl BA, Schubbe M, et al. Histopathology of Anterior Urethral Strictures: Toward a Better Understanding of Stricture Pathophysiology. J Urol 2019;202:748-56.

Cite this article as: Samarska IV, Dani H, Bivalacqua TJ, Burnett AL, Matoso A. Histopathologic and clinical comparison of recurrent and non-recurrent urethral stricture disease treated by reconstructive surgery. Transl Androl Urol 2021;10(10):3714-3722. doi: 10.21037/tau-21-477
13. Santucci RA, Joyce GF, Wise M. Male urethral stricture disease. J Urol 2007;177:1667-74.

14. Zaid UB, Lavien G, Peterson AC. Management of the Recurrent Male Urethral Stricture. Curr Urol Rep 2016;17:33.

15. Blaschko SD, McAninch JW, Myers JB, et al. Repeat urethroplasty after failed urethral reconstruction: outcome analysis of 130 patients. J Urol 2012;188:2260-4.

16. Santucci R, Eisenberg L. Urethrotomy has a much lower success rate than previously reported. J Urol 2010;183:1859-62.

17. Angermeier KW, Rourke KF, Dubey D, et al. SIU/ICUD Consultation on Urethral Strictures: Evaluation and follow-up. Urology 2014;83:S8-17.

18. Yeung LL, Brandes SB. Urethroplasty practice and surveillance patterns: a survey of reconstructive urologists. Urology 2013;82:471-5.

19. Breyer BN, McAninch JW, Whitson JM, et al. Effect of obesity on urethroplasty outcome. Urology 2009;73:1352-5.

20. Breyer BN, McAninch JW, Whitson JM, et al. Multivariate analysis of risk factors for long-term urethroplasty outcome. J Urol 2010;183:613-7.

21. Privratsky JR, Almassi N, Guralnick ML, et al. Outcomes of grafted bulbar urethroplasty in men with class II or III obesity. Urology 2011;78:1420-3.

22. Yalcinkaya F, Zengin K, Sertcelik N, et al. Dorsal onlay buccal mucosal graft urethroplasty in the treatment of urethral strictures - does the stricture length affect success? Adv Clin Exp Med 2015;24:297-300.

23. Levy M, Gor RA, Vanni AJ, et al. The Impact of Age on Urethroplasty Success. Urology 2017;107:232-8. 